\title{
Defective Expression of gp180, a Novel CD8 Ligand on Intestinal Epithelial Cells, in Inflammatory Bowel Disease
}

\author{
Lisa S. Toy, ${ }^{\star \ddagger}$ Xian Yang Yio, ${ }^{*}$ Annica Lin, ${ }^{*}$ Shaun Honig, ${ }^{*}$ and Lloyd Mayer ${ }^{\star}$ \\ *Division of Clinical Immunology and the ${ }^{*}$ Dr. Henry D. Janowitz Division of Gastroenterology, Mount Sinai Medical Center, \\ New York 10029
}

\begin{abstract}
Previous studies support a role for intestinal epithelial cells (IEC) as antigen-presenting cells in mucosal immune responses. $\mathrm{T}$ cells activated by IEC are $\mathrm{CD} 8+$, suppressor in function, and dependent upon CD8-associated p56lck activation. A $180-\mathrm{kD}$ glycoprotein (gp180) recognized by mAbs B9 and L12 has been identified and shown to be important in $\mathrm{CD} 8+\mathrm{T}$ cell activation by IEC. Since IEC derived from patients with inflammatory bowel disease (IBD) are incapable of activating CD8 $+T$ cells, we asked whether this correlated with gp180 expression. While frozen sections of normal bowel revealed bright gp180 staining on all IEC, both inflamed and uninflamed ulcerative colitis (UC) specimens showed patchy staining. In Crohn's disease (CD), staining was faint to absent. Flow cytometry confirmed immunohistochemical data. The staining patterns correlated with the ability of IEC to activate CD8-associated p56lck. Normal IEC induced phosphorylation of p56lck in CD8 $\alpha$ but not CD4+ transfectants. In contrast, both UC and CD IEC activated CD4 and, to a much lesser extent, CD8-associated p56lck. Thus, gp180 expression by IBD IEC appears to be altered, and correlates with a functional alteration of $l c k$ activation. This defect may reflect a more proximal event in the pathogenesis of IBD. (J. Clin. Invest. 1997. 100:2062-2071.) Key words: epithelium $\bullet \mathrm{CD} 8+\mathrm{T}$ cells $\bullet$ Crohn's disease $\bullet$ ulcerative colitis $\cdot$ glycoprotein
\end{abstract}

\section{Introduction}

The inflammatory bowel diseases (IBDs), ${ }^{1}$ ulcerative colitis (UC) and Crohn's disease (CD), are characterized by chronic

Address correspondence to L. Mayer, M.D., Division of Clinical Immunology, Mount Sinai Medical Center, 1 Gustave L. Levy Place, Box 1089, New York, NY 10029. Phone: 212-241-5992; FAX: 212-9875593; E-mail: lmayer@smtplink.mssm.edu

Received for publication 10 October 1996 and accepted in revised form 10 July 1997.

1. Abbreviations used in this paper: $\mathrm{CD}$, Crohn's disease; CDI, inflamed intestine from patients with $\mathrm{CD}$; CDU, uninflamed intestine from patients with CD; DIV, diverticulitis; gp180, 180-kD glycoprotein; IBD, inflammatory bowel disease; IEC, intestinal epithelial cells; NL, normal tissue; SCID, severe combined immunodeficiency; UC, ulcerative colitis; UCI, inflamed intestine from patients with UC; UCU, uninflamed intestine from patients with UC.

J. Clin. Invest.

(C) The American Society for Clinical Investigation, Inc. 0021-9738/97/10/2062/10 \$2.00

Volume 100, Number 8, October 1997, 2062-2071

http://www.jci.org active inflammation in an organ where controlled inflammation is the norm. Work from several laboratories has supported the concept that this active inflammatory process is the result of a dysregulated immune response. More specifically, there is evidence of constitutively activated CD4+ T cells within the lamina propria that can leak into the systemic circulation $(1,2)$. These activated CD4+ $\mathrm{T}$ cells secrete a panel of cytokines that may promote localized cell-mediated granulomatous inflammation (CD) (3-6) or a more diffuse antibodyand complement-mediated Arthus-type reaction (UC) $(7,8)$. While many pathogenetic mechanisms have been proposed to explain these observations, to date no consistent infectious, neurohormonal, or immunologic mechanism has been elucidated. Despite this, there have been tremendous advances in our knowledge of these diseases. There is a growing consensus that IBD reflects the combination of an altered immune response to one of multiple luminal antigens in a genetically susceptible host. The nature of the altered immune response, the aberrant activation of CD4+ $\mathrm{T}$ cells in the lamina propria, remains to be determined.

Our laboratory has long been interested in the role of intestinal epithelial cells (IEC) in regulating mucosal immune responses. In vitro studies suggest that normal epithelial cells can activate CD8+ suppressor $\mathrm{T}$ cells, which may be relevant to the controlled inflammation seen in the intestine in vivo $(9$, 10). We have identified previously a molecule expressed on normal IEC that appears to play a role in the activation of antigen nonspecific CD8+ suppressor T cells $(11,12)$. This molecule, recognized by two mAbs, B9 and L12, is a $180-\mathrm{kD}$ glycoprotein (gp180) that mediates binding to CD8 and results in the activation of the src-like tyrosine kinase p56lck (13). Activation of this kinase is necessary but not sufficient for the proliferation of CD8+ T cells induced by normal epithelial cells.

Previous studies from this laboratory have shown that there is a defect in the activation of suppressor cells by IEC derived from patients with IBD (14). These results are seen regardless of the site from which the epithelial cells are derived. That is, epithelial cells from both actively inflamed and uninvolved areas of IBD display similar defects. This suggests that there may be an inherent defect in all IBD epithelium that results in the failure of suppressor cell generation. When IBD epithelial cells are called upon to present antigen, they may do so in an aberrant fashion, resulting in the activation of CD4+ $\mathrm{T}$ cells with consequent chronic active inflammation. Nonimmune physical and chemical barriers, such as tight junctions and intestinal mucus, may prevent chronic inflammation from being a constant in these patients.

In this study, we show that defects in gp180 expression are found in IEC from UC and CD, and correlate with functional differences in the activation of CD8+ suppressor T cells. We hypothesize that the alterations in gp180 expression may lead potentially to a loss in the capacity of IBD IEC to mediate suppressor cell activation, allowing for the inflammation characteristic of IBD to persist. 


\section{Methods}

Specimens. Surgical specimens from patients undergoing bowel resection for cancer (10 cm away from tumor), CD, UC, or other inflammatory diseases [diverticulitis (DIV), ischemic colitis] at the Mount Sinai Medical Center were used as a source of epithelial cells. For flow-cytometric studies, endoscopic biopsies from normal and IBD patients undergoing diagnostic or surveillance colonoscopy were also used as epithelial cell sources. Samples were obtained from 16 normal patients (histologically and grossly normal areas of intestinal specimens derived from patients having colonoscopy or bowel resection for cancer and non-IBD inflammatory conditions) and 48 IBD patients. The presence or absence of inflammation in tissues was determined grossly and confirmed histologically.

Records of all patients were reviewed, and their histories and medical regimens noted. None of the patients were on high-dose immunomodulators (6-mercaptopurine, azathioprine) at the time of surgery. Their treatment regimens included 5-aminosalicylic acid alone, steroids alone, 6-mercaptopurine and steroids, or no therapy at all.

$m A$ bs. mAbs B9 and L12 (both IgG1) against human intestinal epithelial cell membrane antigens were generated in our laboratory as previously described (11). Both antibodies recognize different carbohydrate side chains on gp180. This molecule appears to be involved in the activation of CD8 + T cells by normal IEC (12). Positive and negative controls for IEC staining used mAbs W6/32 (American Type Culture Collection, Rockville, MD) and IgG1 antidinitrophenol (the kind gift of Dr. Jay Unkeless, Mount Sinai Medical Center, New York), respectively.

Immunohistochemical studies. Tissue sections were stained by a well-described method (15). Bowel specimens were placed in cold calcium and magnesium-free HBSS (CMF HBSS; GIBCO BRL, Gaithersburg, MD) at the time of surgery and transported immediately to the laboratory. They were then oriented and snap-frozen in Tissue Tek (Miles Scientific Div., Miles Laboratories, Inc., Naperville, IL) and either stored at $-70^{\circ} \mathrm{C}$ or processed immediately for thin sectioning. Serial 4- $\mu \mathrm{m}$-thick frozen sections were prepared and stored at $-70^{\circ} \mathrm{C}$ for staining. Indirect staining with B9 and L12 mAbs was performed using an avidin-biotin-peroxidase technique (ABC-Vectastain Kit; Vector Laboratories, Inc., Burlingame, CA), with Gill's hematoxylin (Fisher Scientific Co., Springfield, NJ) as a counterstain. All stained sections were reviewed blindly and independently by at least two investigators, including a trained pathologist. Discrepancies in interpretation were resolved by review of the slides by both investigators and at least one additional investigator. Criteria for positive staining were set before initiation of the studies and agreed to by all investigators. As a positive control, staining with the anti-class I mAb W6/32 was performed in each series of experiments. Each section was compared with a corresponding control consisting of an irrelevant isotypematched antibody or no added antibody. No significant background staining was evident.

Isolation of epithelial cells. The procedure used to isolate epithelial cells has been described previously (16). Resected tissue specimens were rinsed extensively with PBS to remove loosely adherent material, and then rubbed gently with sterile gauze. The surface mucosa was stripped off from the underlying submucosa by careful dissection and minced into tiny pieces. These were then washed several times in RPMI 1640 containing 1\% penicillin/streptomycin, 1\% fungizone, $50 \mathrm{mg} / \mathrm{ml}$ gentamicin (all from GIBCO BRL) and $10 \mathrm{mM}$ Hepes (Sigma Chemical Co., St. Louis, MO). The tissue was then treated with $1 \mathrm{mM}$ DTT (Sigma Chemical Co.) in RPMI 1640 for 15 min at $22^{\circ} \mathrm{C}$. After washing three times with RPMI 1640 containing antibiotics, the tissue pieces were incubated in RPMI 1640 containing $1 \mathrm{mg} / \mathrm{ml}$ dispase (Boehringer Mannheim Biochemicals, Indianapolis, IN) for $30 \mathrm{~min}$ at $37^{\circ} \mathrm{C}$ in an orbital shaker rotating at $150 \mathrm{rpm}$. During this treatment, epithelial cells and intraepithelial lymphocytes were released from the tissue. Cells that remained loosely adherent to the tissue were recovered using a rubber policeman. Dispase treatment was repeated once. The cell suspensions resulting from dispase treatments were washed twice, pelleted, and resuspended in a $15-\mathrm{ml}$ conical tube in $3 \mathrm{ml}$ of $100 \%$ Percoll (Pharmacia Biotech, Piscataway, $\mathrm{NJ}$ ) which had been adjusted to $\mathrm{pH} 7.4$ with an osmolality of 290 mosM. 3-ml layers of 60, 40, and 30\% Percoll (prepared by diluting $100 \%$ Percoll with RPMI 1640) were layered successively on top to establish a Percoll density gradient. The Percoll gradient was centrifuged at $1,500 \mathrm{rpm}$ for $30 \mathrm{~min}$ at $4^{\circ} \mathrm{C}$. Cells at the top $0 / 30 \%$ layer interface contained $>95 \%$ pure epithelial cells. Purity was confirmed by flow-cytometric analysis (PROFILE; Coulter Corp., Hialeah, FL) using anti-CD14, -CD3, -CD20 (Coulter Corp.), and L12 (anti-epithelial cell) $\mathrm{mAbs}$. Contaminating cells were predominantly $\mathrm{CD} 3+\mathrm{T}$ cells $(2-4 \%)$. Cells were washed three times in RPMI, and viability was determined by trypan blue exclusion. Only cell preparations with a viability $>90 \%$ were used for staining and analysis in T cell-IEC cocultures. Preliminary studies using colonic epithelial cell lines documented that dispase treatment had no effect on the expression of gp180 as assessed by staining with mAbs B9 and L12.

Quantitation of gp180 in IEC. $10^{5}$ freshly isolated IEC were washed three times with $1 \%$ BSA (Sigma Chemical Co.) in PBS, then incubated for 45 min with $\mathrm{mAbs}$ B9 or L12 previously conjugated to FITC (Sigma Chemical Co.) by a well-established method (17). Next, the cells were washed three times with $1 \%$ BSA-PBS and then analyzed by flow cytometry. Positive and negative controls for IEC staining used $\mathrm{mAbs} \mathrm{W} 6 / 32$ and $\mathrm{IgG} 1$ antidinitrophenol, which had also been conjugated to FITC.

To further ensure the purity of our IEC preparation, two sets of analyses were performed. First, to eliminate possible lymphocyte contamination, IEC preparations were also incubated with KC56 (T-200)-RD1 (anti-CD45 mAb conjugated to phycoerythrin; Coulter Corp.). CD45+ cells were gated out during flow cytometry. In the second analysis, IEC preparations were permeabilized using PermeaFix (Ortho Diagnostic Systems Inc., Raritan, NJ) and incubated with CAM 5.2 (anti-human cytokeratin mAb; Becton Dickinson, San Jose, CA) followed by goat anti-mouse Ig conjugated to R-phycoerythrin (Biosource International, Camarillo, CA). During flow cytometry, measurements of gp180 expression were determined only on cells positive for CAM 5.2. This second approach was more time-consuming than the first, but both approaches yielded similar data.

Preparation of IEC lysates. $10^{6}$ freshly isolated epithelial cells were incubated in an Eppendorf tube on ice with cold lysis buffer (1\% NP-40, $1 \mathrm{mM}$ PMSF, $10 \mathrm{mM}$ iodacetamide, and $1 \mathrm{mM} \mathrm{Na} \mathrm{VO}_{4}$ [all from Sigma Chemical Co.], in four parts PBS to one part distilled water) for $30 \mathrm{~min}$, vortexing every $5 \mathrm{~min}$. The lysate was centrifuged at $13,000 \mathrm{rpm}$ for $10 \mathrm{~min}$ in a microfuge to remove cell debris.

Murine transfectants $3 G 4$ and $3 G 8$. The transfectants $3 \mathrm{G} 4$ and $3 \mathrm{G} 8$ are murine T cell hybridomas expressing human CD4 and CD8 $\alpha$ antigens, respectively. They were kindly donated by Dr. S.J. Burakoff (Dana-Farber Cancer Institute, Boston, MA). Construction of these transfectants has been detailed previously. To produce the $3 \mathrm{G} 4$ cell line, the T cell hybridoma BY155.16 was transfected with the defective retrovirus MNST4 carrying full-length human CD4 cDNA, and murine transfectants expressing human CD4 antigen were selected by sorting (18). The $3 \mathrm{G} 8$ cell line was constructed in a similar manner, using the T cell hybridoma BY155.16 and the retroviral expression vector MNCT8 carrying full-length human CD8 $\alpha$ cDNA (19).

Mixed T cell-IEC coculture. $0.5-1 \times 10^{6} 3 \mathrm{G} 4$ or $3 \mathrm{G} 8$ cells were incubated in an Eppendorf tube with equal numbers of normal or IBD IEC or antibodies cross-linking CD4 or CD8 in a $37^{\circ} \mathrm{C}$ water bath for varying time periods. At specific time points, the tube was transferred to ice, and $1 \mathrm{ml}$ of ice-cold stop buffer $\left(1 \mathrm{mM} \mathrm{Na}_{3} \mathrm{VO}_{4}\right.$ in PBS) was added. The cells were then pelleted, and $100 \mathrm{ml}$ of ice-cold lysis buffer (1 mM PMSF, $10 \mathrm{mM}$ iodacetamide, and $1 \mathrm{mM} \mathrm{Na}_{3} \mathrm{VO}_{4}$, in four parts PBS to one part distilled water) was added for $30 \mathrm{~min}$, vortexing every $5 \mathrm{~min}$. Next, the lysates were centrifuged at 13,000 rpm for $1 \mathrm{~min}$ in a microfuge to remove cell debris.

Western blot with $B 9$ or antiphosphotyrosine $m A b s$. IEC or mixed T cell-IEC lysates were resolved on $10 \%$ SDS-PAGE and transferred onto a nitrocellulose membrane at $15 \mathrm{~V}$ overnight in transfer buffer (20\% methanol, $150 \mathrm{mM}$ glycine, $25 \mathrm{mM}$ Tris, $\mathrm{pH} 8.3$ ). The transfer 
sandwich was prewetted in transfer buffer and set up in the following order: $(a)$ a porous polyethylene foam sheet; $(b)$ one sheet of $3 \mathrm{M}$ filter paper; $(c)$ the gel; $(d)$ a nitrocellulose membrane sheet $(0.2 \mu \mathrm{m})$ (Schleicher \& Schuell, Inc., Keene, NH); $(e)$ another sheet of 3M filter paper; and $(f)$ another sheet of porous polyethylene foam. The transfer sandwich was inserted into a transfer electrophoresis unit (Hoefer Scientific Instruments, San Francisco, CA) with the nitrocellulose membrane at the anode. After transfer, the nitrocellulose sheet was blocked by $50 \mathrm{ml}$ of $5 \%$ nonfat milk in PBS. The nitrocellulose sheet was washed once with PBS and transferred to a plastic bag containing B9 $\mathrm{mAb}(10 \mu \mathrm{g} / \mathrm{ml})$ for IEC lysates or $4 \mathrm{G} 10$ antiphosphotyrosine $\mathrm{mAb}(0.5 \mu \mathrm{g} / \mathrm{ml})$ for $\mathrm{T}$ cell-IEC cocultures at $4^{\circ} \mathrm{C}$ overnight. The sheet was washed five times with PBS for $5 \mathrm{~min}$ each, and then transferred to a new plastic bag containing $0.5 \mu \mathrm{g} / \mathrm{ml}$ goat anti-mouse IgG conjugated with horseradish peroxidase (Organon Teknika-Cappel, West Chester, PA). The incubation was performed at room temperature for $2 \mathrm{~h}$. The sheet was then washed five times with washing buffer $(0.05 \%$ Tween 20 in PBS) for 5 min each, and finally incubated with $12 \mathrm{ml}$ of chemiluminescence reagent (DuPont, Wilmington, DE) at room temperature for $1 \mathrm{~min}$. A sheet of plastic wrap was placed onto the nitrocellulose sheet, and the sheet was exposed and developed (XAR-5 film; Eastman Kodak Co., Rochester, NY).

\section{Results}

Expression of gp180 is reduced in IBD. Immunohistochemical staining using anti-gp180 mAbs B9 and L12 revealed striking differences between tissues derived from normal intestine and those from IBD specimens. As seen in Fig. $1 A$, staining of normal mucosa with mAb B9 revealed the presence of gp180 in both surface and crypt epithelium (surface $>$ crypt), with both an apical and basolateral distribution. At higher magnification, one can see intense apical staining with more evenly distributed basolateral staining (Fig. $1 \mathrm{~B}$ ). Studies from our laboratory have shown that the apical staining in cell lines is reduced after treatment with phosphatidylinositol-specific phospholipase $\mathrm{C}$ while the basolateral staining is maintained. These findings suggest that the apically sorted form of gp180 is glycosylphosphatidylinositol-anchored, whereas the basolateral form is likely to be transmembrane (12). The staining of epithelial cells by mAb B9 from normal controls was comparable regardless of the site (cecum; ascending, transverse, descending, or sigmoid colon; rectum; or ileum) from which the IEC were isolated (see below, and data not shown).
A

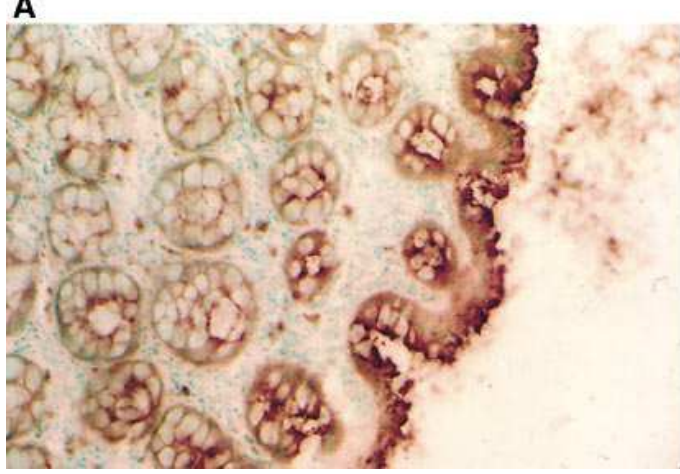

C

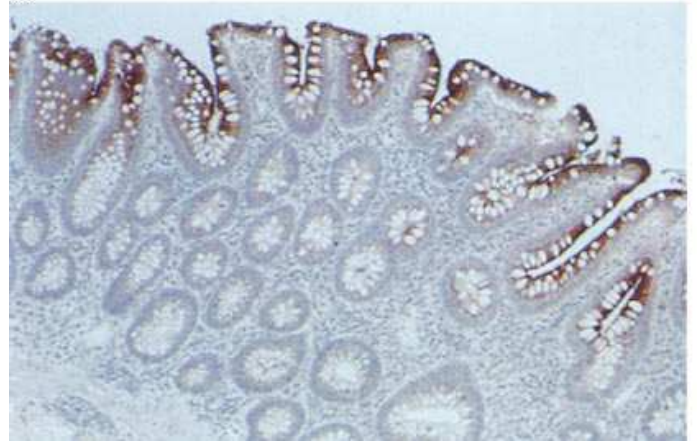

E

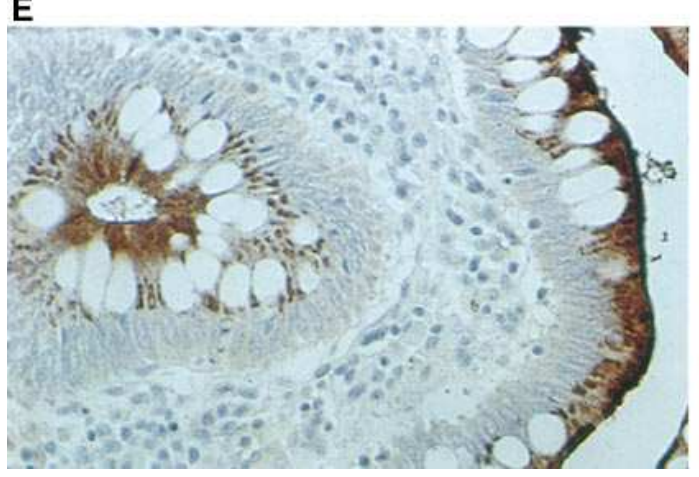

B

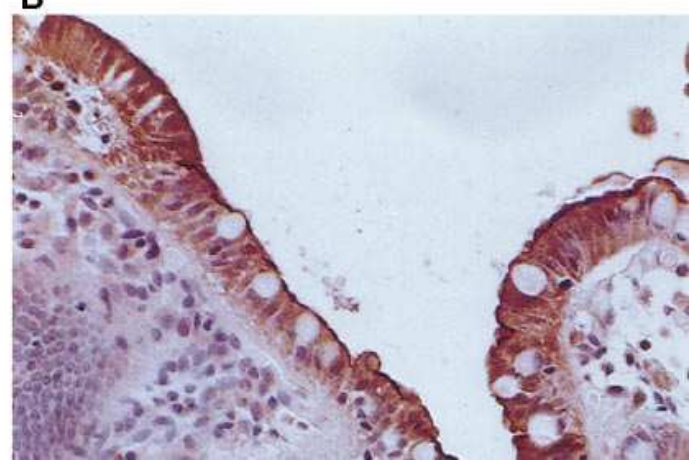

D

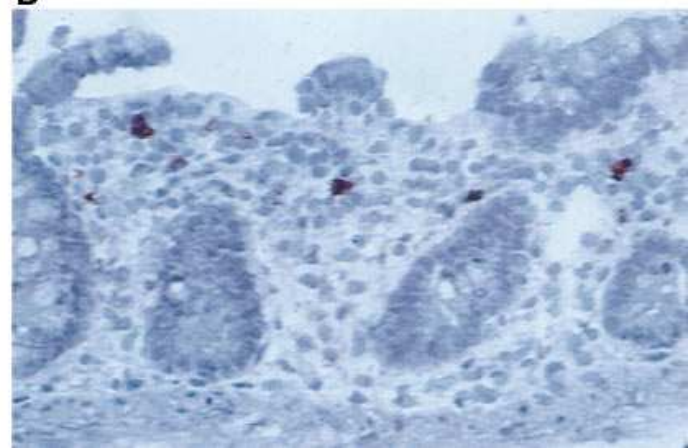

$\mathbf{F}$

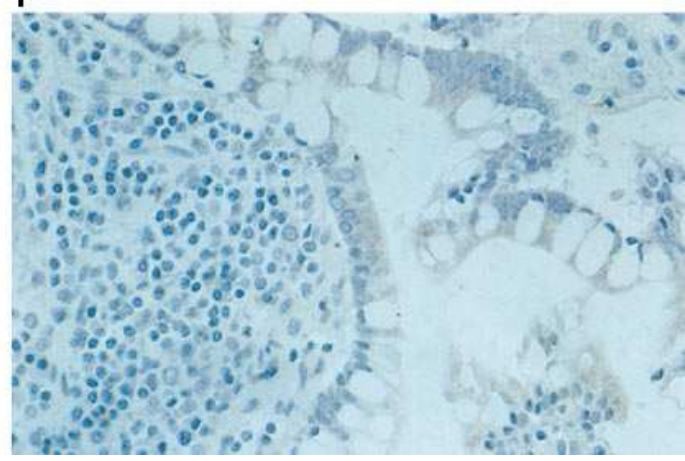

Figure 1. Immunohistochemical staining of frozen sections from $(A)$ normal intestinal tissue using anti-gp180 mAb B9 at low magnification $(\times 10)$ and $(B)$ higher magnification $(\times 20)$; a UC intestinal specimen using anti-gp $180 \mathrm{mAb} \mathrm{B} 9$, $(C)(\times 10)$ and $(D)(\times 20)$ showing directly adjacent areas, and $(E)$, high power magnification $(\times 40)$ of staining area; and $(F)$ an uninflamed region of a $C D$ intestinal specimen using antigp180 mAb B9 $(\times 20)$. Fresh tissue sections were obtained at the time of surgery and snap-frozen immediately in Tissue Tek. Serial $4-\mu \mathrm{m}$-thick frozen sections were cut, and indirect staining with $\mathrm{mAb}$ B9 was performed using an avidin-biotinperoxidase technique, with Gill's hematoxylin as a counterstain. 


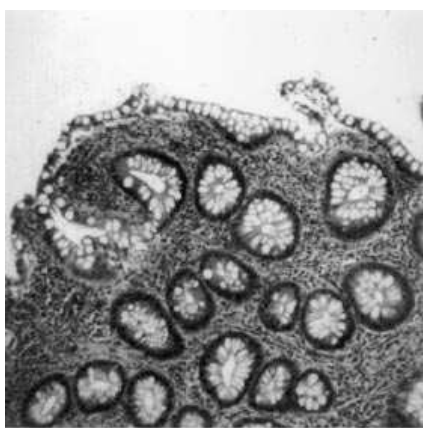

A

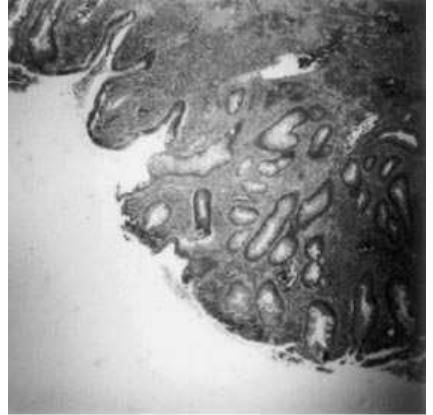

B

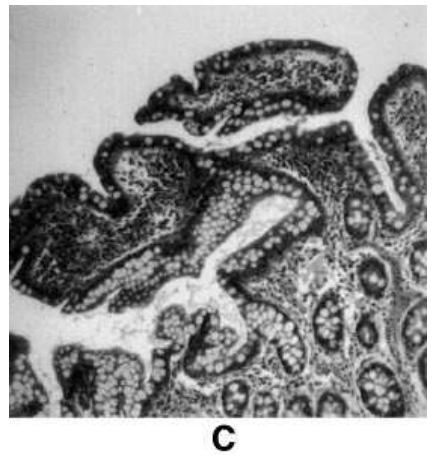

Figure 2. Hematoxylin and eosin staining of frozen sections from IBD intestinal specimens. $(A)$ Inflamed $(\times 10)$ and $(B)$ uninflamed $(\times 10)$ areas of colon from the same UC patient shown in Fig. 1, $C-E$. (C) Moderately inflamed $(\times 10)$ area from the same $C D$ patient shown in Fig. $1 F$.
In contrast to these findings, staining with both mAbs B9 and L12 in bowel sections derived from patients with UC revealed a more patchy distribution, some areas staining with normal intensity, while other adjacent areas showed no staining at all (Fig. 1, $C$ and $D$; hematoxylin and eosin-stained sections from the same patient are seen in Fig. 2, $A$ and $B$ ). However, even in the mAb B9-stained areas, the pattern of staining was altered. There appeared to be a loss of basolateral staining, with preservation or even enhancement of apical gp180 (Fig. $1 E$ ). These changes were noted both in areas of active inflammation and in areas where there was no gross or histologic disease (e.g., ileum and right colon). Significantly, this alteration in gp180 expression was observed consistently in all intestinal tissues derived from UC patients, regardless of their history and medical regimen (including patients on no medical therapy at all).

The difference in staining of epithelial cells with mAbs B9 and L12 in patients with CD was even more dramatic. In tissues obtained from both inflamed and uninvolved regions of bowel, little to no staining with either mAb B9 or L12 was noted (Fig. $1 \mathrm{~F}$; the immunohistochemically stained section is from an uninflamed region. Hematoxylin and eosin-stained sections from the same patient are seen in Fig. $2 C$ ). The marked decrease in staining was seen in all intestinal tissues derived from patients with CD, in inflamed and uninflamed areas. As seen in UC, there was no correlation of staining patterns with any medical regimen. Of note, the pattern of gp180 expression seen in CD (uniformly decreased B9 staining) differs from that observed in UC (patchy and more apical, less basolateral B9 staining). These findings suggest a difference between $\mathrm{CD}$ and $\mathrm{UC}$; in the former, a more global deficiency in gp180 expression exists, whereas in UC, there appears to be a reduction as well as an intracellular redistribution of the molecule. Clearly, the uniform defect in $\mathrm{CD}$ and the patchy defect seen in UC do not explain the typical tissue distribution of each of these disorders. Other factors must be playing a role in the site of disease expression (see Discussion).

Quantitation of gp180 in normal and IBD IEC. To confirm the immunohistochemical studies in a quantitative manner, isolated IEC stained with mAbs B9 and L12 were analyzed by flow cytometry (Fig. $3 A$ ). Normal IEC stained in a uniform distribution, with a fluorescence intensity slightly less than that seen by staining with $\mathrm{W} 6 / 32$, an $\mathrm{mAb}$ to class I MHC. IEC derived from inflamed non-IBD tissues (DIV, ischemic colitis) revealed a staining pattern similar to normal controls. However, IEC from UC tissues showed a bimodal pattern of staining: one population with a fluorescence intensity similar to that seen in normal IEC, and one nonstaining population. This result correlates with the patchy staining seen in the immunohistochemical studies of UC mucosa. The striking decrease in staining in CD IEC observed in immunohistochemical studies was also observed by flow cytometry. IEC from CD demonstrated very little or no staining with mAb B9 or L12. As in the immunohistochemical studies, no correlation between medical regimen and gp180 expression was found. In addition, IEC were gated for selectively by concurrent staining with the anticytokeratin antibody CAM 5.2 (Fig. 3 B). Similarly, CD45+ contaminating lymphocytes were gated out in these analyses (data not shown). Thus, the differences in staining reflected differences in the IEC and not in contaminating populations.

Notably, IEC derived from both actively inflamed and uninflamed IBD tissues revealed similar patterns of staining, again suggesting that inflammation in itself is not responsible for the differences seen between normal intestine and IBD specimens. Interestingly, although the overall pattern of staining remained the same, some upregulation of staining with mAb B9 or L12 was observed in the actively inflamed IBD tissues, when compared with uninflamed areas from those same specimens. These findings are consistent with preliminary experiments in our laboratory which demonstrate that gp180 can be upregulated by IFN- $\gamma$ and GM-CSF, cytokines present in increased amounts in IBD mucosa (20).

When all samples were analyzed together [normal tissue (NL), inflamed and uninflamed UC (UCI and UCU), and inflamed and uninflamed CD (CDI and CDU) IEC groups], clear patterns emerged. Whereas staining of NL control IEC with $\mathrm{mAb} B$ 9 revealed a wide scatter of fluorescence intensities, the staining intensities of the CDU, CDI, and UCU groups were significantly different from that of the NL group when compared by Student's $t$ test (Fig. 4; NL vs. CDU, $P<0.003$; NL vs. CDI, $P<0.01$; and NL vs. UCU, $P<0.04)$. Although not significantly different $(P<0.14)$, the staining by mAb B9 within the UCI group tended to be lower than that seen in the NL group.

The differences in gp180 expression might relate to the possibility that there is a defect in transporting this glycoprotein to the surface in IBD IEC. While this was less likely, given the absence of staining in fixed immunohistochemical sections, we chose to assess this possibility by analyzing total gp180 by mAb B9 Western blot analysis of IEC lysates. The intensity of the $180-\mathrm{kD}$ band in both uninflamed UC and CD IEC was markedly reduced or absent when compared to the band in NL IEC (Fig. 5). Thus, the failure to express gp180 in IBD tissues 

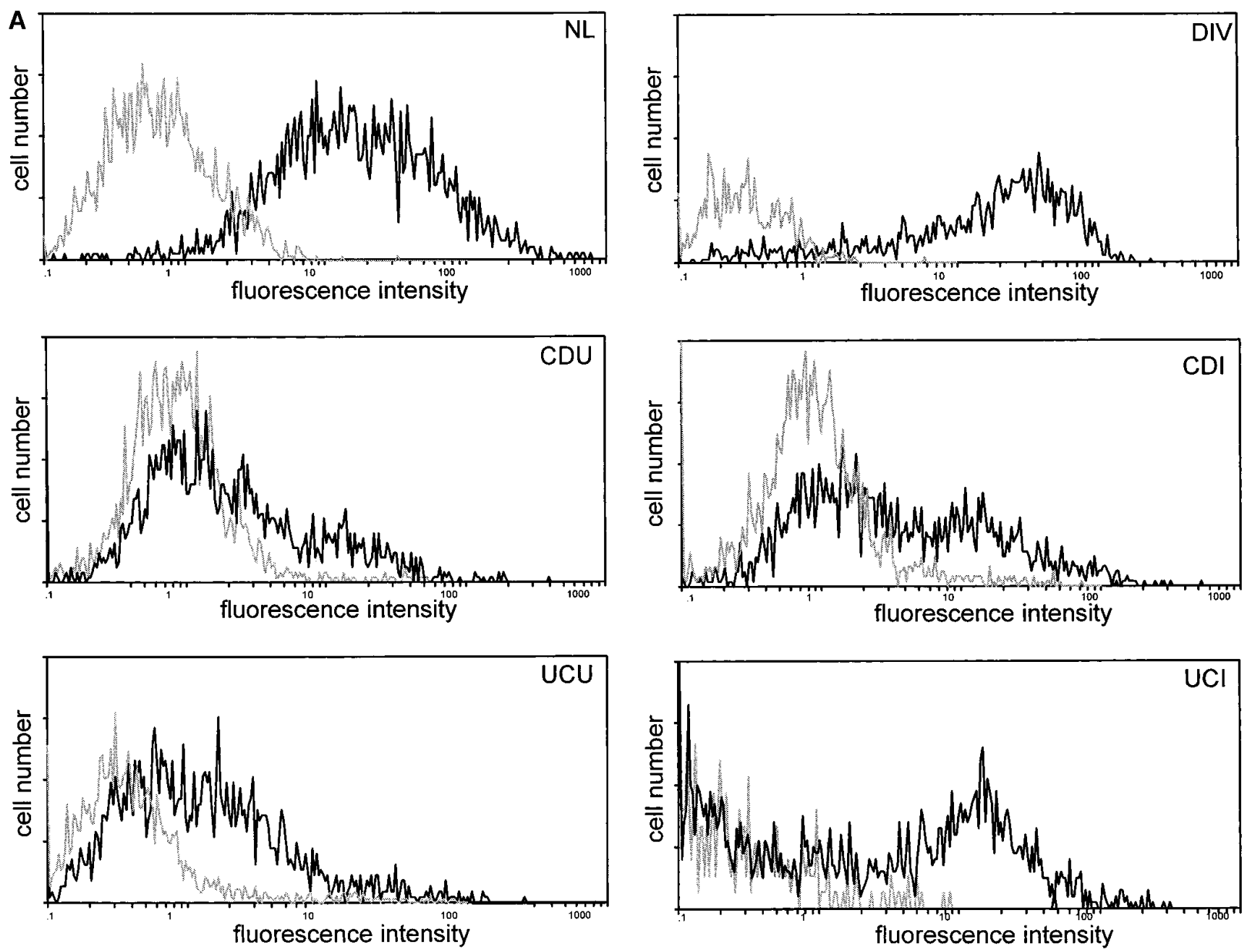

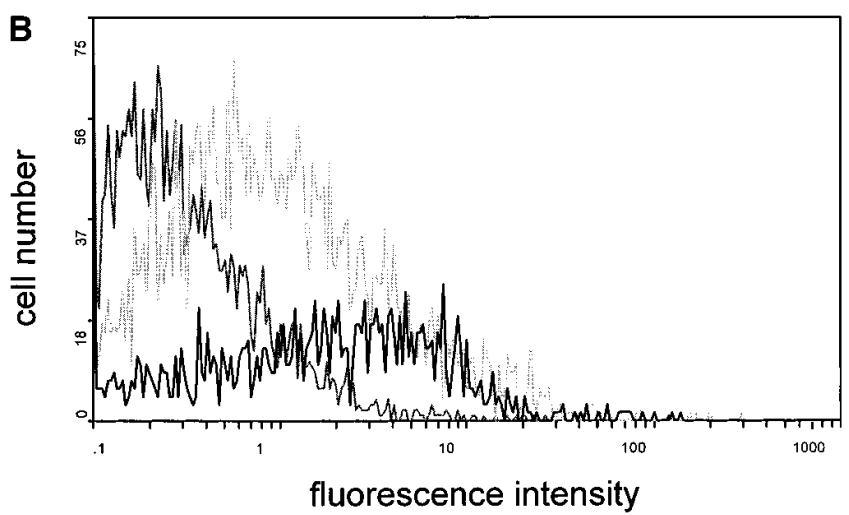

appears to result from reduced production of the form recognized by these mAbs.

Functional consequences of reduced gp180 expression. We next wanted to determine whether this alteration in gp180 expression correlated with a functional phenotype. As described previously (14), the selective stimulation of CD8+ T cells by NL IEC appears to require the activation of a src-like tyrosine kinase, p56lck, associated with the intracytoplasmic tail of the $\mathrm{CD} 8 \alpha$ chain. Activation of p56lck results in autophosphorylation as well as phosphorylation of associated substrates. In-
Figure 3. (A) Flow-cytometric analysis of mAb B9 staining of IEC freshly isolated from NL, DIV, CDI, CDU, UCI, and UCU specimens, compared with staining by negative control IgG1. Surface mucosa was dissected from fresh tissue specimens, treated with $1 \mathrm{mM}$ DTT in RPMI, and then incubated with $1 \mathrm{mg} / \mathrm{ml}$ dispase in order to release IEC and intraepithelial lymphocytes. IEC were further purified by Percoll density gradient centrifugation. IEC were then stained with $\mathrm{mAb}$ $\mathrm{B} 9$ (dark line), IgG1 (light line), and $\mathrm{W} 6 / 32$ (anti-class I mAb, a positive control; not shown) and analyzed by flow cytometry. $x$ axis, Mean fluorescence intensity. $y$ axis, Relative cell numbers. (B) Flow-cytometric analysis of $\mathrm{mAb}$ B9 and anti-human cytokeratin antibody CAM 5.2 staining of IEC freshly isolated from an NL specimen. IEC were treated as in $A$, then stained with mAb B9 (light line), CAM 5.2 (dark line), IgG1 (medium line), and W6/32 (not shown). $x$ axis, Mean fluorescence intensity. $y$ axis, Relative cell numbers.

hibition of this kinase inhibits CD8+ T cell activation by NL IEC. Furthermore, addition of the anti-gp180 mAb B9 to T cell-IEC cocultures inhibits the activation of p56lck, whereas anti-class I and anti-class II mAbs do not. To define more carefully specific pathways involved in T cell-IEC interactions, we used murine $\mathrm{T}$ cell hybridomas transfected with either fulllength human CD4 cDNA (3G4) or full-length CD8 $\alpha$ cDNA (3G8). The only human proteins expressed on these cells are either CD4 or CD8. Others have shown that murine p56lck can associate with either of these molecules $(18,19)$. Cross- 


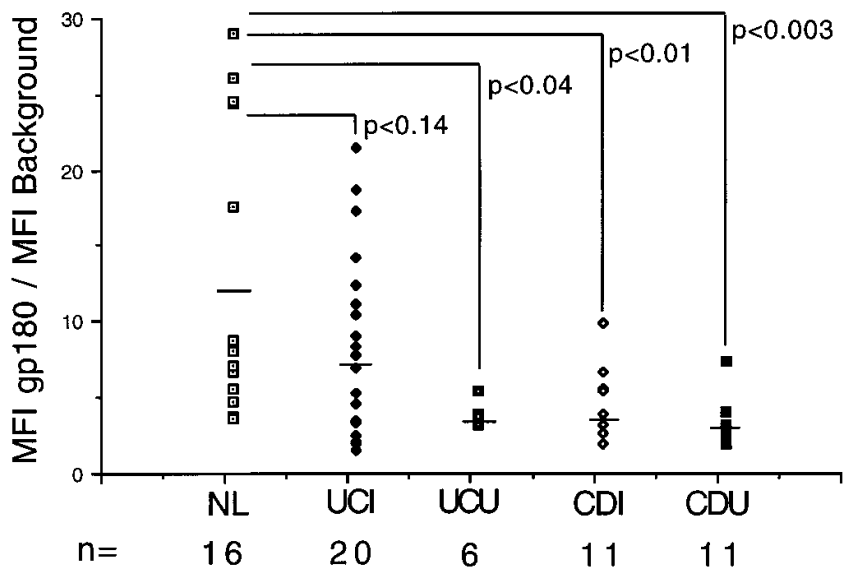

Figure 4. Mean fluorescence intensities of mAb B9 staining in samples of freshly isolated IEC, compared with background, by group. IEC were isolated and treated as described in Fig. 2. Short lines depict the mean values for each group.

linking of CD4 on $3 \mathrm{G} 4$ with anti-CD4 mAbs, or cross-linking CD8 on $3 \mathrm{G} 8$ with anti-CD8 mAbs, will result in the autophosphorylation of p56lck and associated substrates. Thus, activation of p56lck in these transfectants by human IEC can occur only via binding of CD4 or CD8, and each cell line serves as a control for the other.

The results of mixed T cell-IEC coculture assays using 3G4 and 3G8 $\mathrm{T}$ cells are shown in Figs. 6-8. As described previously, when NL IEC are cocultured with 3G4 cells, no upregulation of p56lck is seen when compared with baseline at 0 min (Fig. 6 A) (13). In contrast, p56lck is activated in 3G8-IEC cocultures at 1-5 min, supporting the concept that IEC express a molecule capable of binding to CD8, allowing for kinase activation (Fig. $6 \mathrm{~B}$ ). In multiple experiments, the increase in CD8-associated p56lck was between 3- and 10-fold. Importantly, neither blocking anti-class I nor anti-class II mAbs can inhibit this activation event. Only antibody to gp180 (mAb B9) can inhibit the activation of p56lck in this system (13). The in-

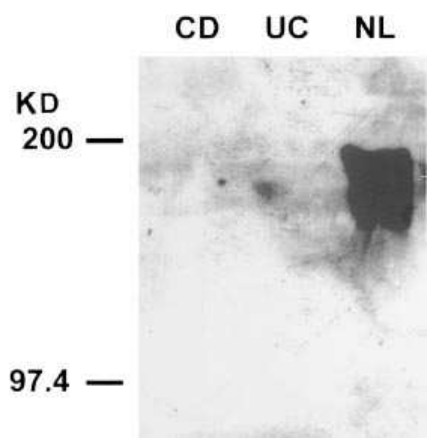

Figure 5. mAb B9 Western blot analysis of IEC lysates from NL and uninflamed bowel derived from patients with CD and UC. $10^{6}$ freshly isolated IEC per group were incubated in lysis buffer. Cell lysates were then resolved on $10 \%$ SDS-PAGE and transferred onto a nitrocellulose membrane. The membrane was blocked with $5 \%$ nonfat milk in PBS, incubated over-

night with $\mathrm{mAb}$ B9, and finally incubated with goat anti-mouse IgG conjugated with horseradish peroxidase. The presence of gp180 was determined by enhanced chemiluminescence.

crease in the baseline phosphorylation in 3G8 cells appears to reflect the dimerization of $\mathrm{CD} 8 \alpha$ chains but does not affect the response of these cells to CD8 ligation.

Since IEC derived from IBD tissues induce the proliferation of CD4 $+\mathrm{T}$ cells but not CD8 $+\mathrm{T}$ cells, we asked whether this finding correlated with a different pattern of p56lck activation. Freshly isolated IEC from both inflamed and uninflamed areas of the colon from a patient with UC were cocultured with $3 \mathrm{G} 4$ and $3 \mathrm{G} 8$ cells. In contrast to cocultures with NL IEC, UC IEC enhanced phosphorylation of p56lck in 3G4 cells (Fig. 6 A). IEC from both inflamed and uninflamed tissues activated CD4-associated $l c k$, although the kinetics of activation were slightly different. Peak activation of CD4-associated p56lck by UCU IEC occurred at $10 \mathrm{~min}$, while UCI IEC strongly activated-CD4 associated p56lck at $1 \mathrm{~min}$. This correlates with the known increase in class II MHC expression on IEC from inflamed areas. Class II antigen binding to CD4 activates CD4associated p56lck. When 3G8 cells were incubated with UCU IEC, no upregulation of CD8-associated p56lck was seen. However, in cocultures with UCI IEC, there was a modest but evident increase in the phosphorylation of CD8-associated lck at 1 min (a roughly twofold increase in signal), which was less than that induced by NL IEC. This suggests that CD8 ligation
A

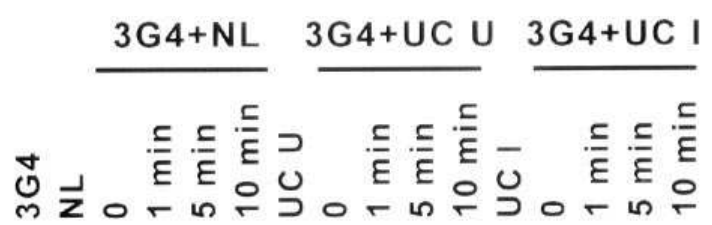

KD

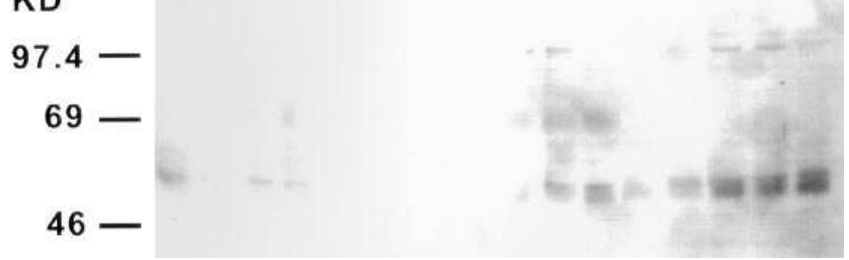

B
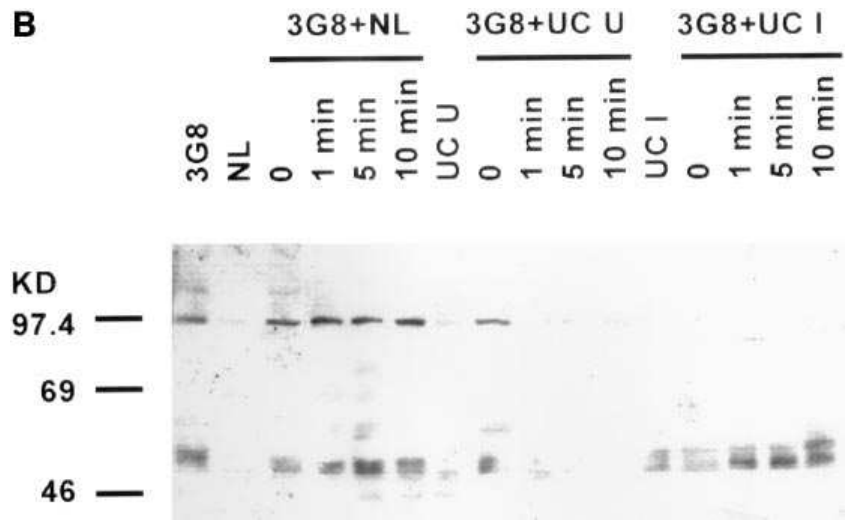

Figure 6. Antiphosphotyrosine Western blot analysis of cell lysates from mixed T cell-IEC cocultures. $(A) 3 \mathrm{G} 4$ or $(B) 3 \mathrm{G} 8$ cells were cocultured with NL, UCU, or UCI IEC for $0,1,5$, or $10 \mathrm{~min}$. Stop buffer was added immediately, and the T cells were lysed with lysis buffer. The lysates were resolved on $10 \%$ SDS-PAGE and transferred onto nitrocellulose membranes. The membranes were blocked with $5 \%$ nonfat milk in PBS, incubated overnight with 4G10 antiphosphotyrosine mAb, and finally incubated with goat anti-mouse IgG conjugated with horseradish peroxidase. Activation of p56lck was determined by enhanced chemiluminescence. Negative control lanes consisting of 3G4 cells, 3G8 cells, and NL, UCU, and UCI IEC alone are also shown. 
A

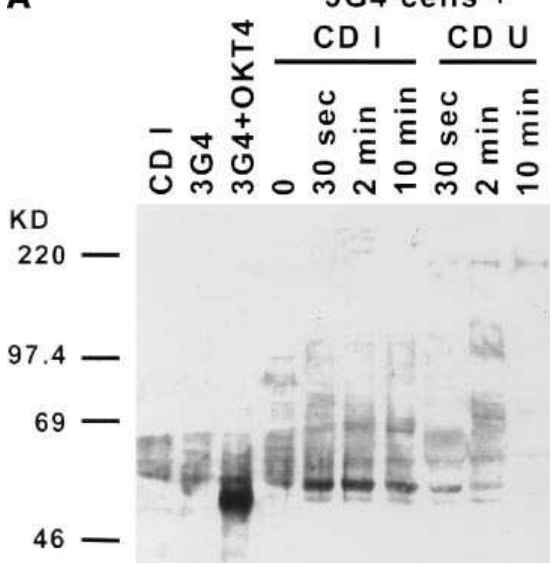

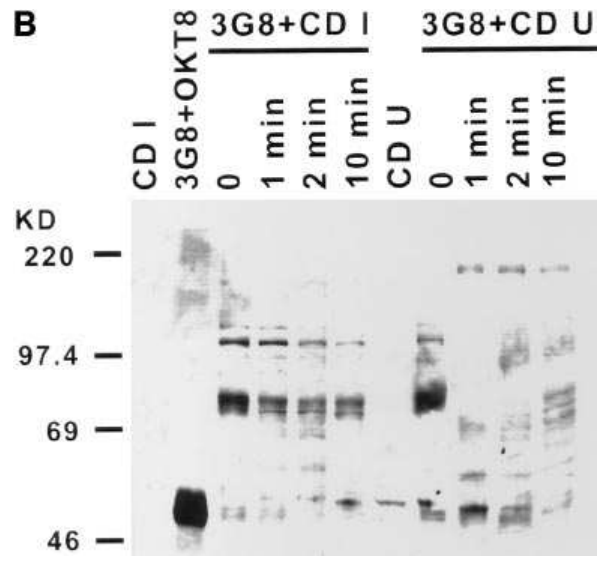

Figure 7. Antiphosphotyrosine Western blot analysis of cell lysates from mixed T cell-IEC cocultures. ( $A$ ) $3 \mathrm{G} 4$ or $(B) 3 \mathrm{G} 8$ cells were cocultured with CDU or CDI IEC for $0,0.5,1,2$, or $10 \mathrm{~min}$. Cell lysates were prepared, transferred to nitrocellulose membranes, and the membranes were treated as described in Fig. 5. Activation of p56lck was determined by enhanced chemiluminescence. Negative controls consisting of $3 \mathrm{G} 4$ cells and CDI and CDU IEC alone are also shown. Positive controls showing p56lck activation when incubating $3 \mathrm{G} 4$ cells with anti-CD4 $\mathrm{mAb}$ (OKT4) and $3 \mathrm{G} 8$ cells with anti-CD8 mAb $(O K T 8)$ are demonstrated as well. may be occurring with UC IEC but that the interaction is weaker. These findings correlate with the present but weaker expression of gp180 on UCI IEC (CD8-associated p56lck activation) as well as the enhanced expression of MHC class II molecules on these cells (CD4-associated p56lck activation).

Like UC IEC, IEC from patients with CD activated p56lck in 3G4 cells (Fig. 7 A). However, once again, CD4-associated lck was activated more by the inflamed than the uninflamed IEC. Maximum activation by CDU and CDI IEC both occurred at $30 \mathrm{~s}$ in 3G4-CD IEC cocultures. Cocultures with 3G8 showed limited CD8-associated lck upregulation for both CDI and CDU IEC, when compared with baseline (Fig. 7 B). These results suggest that $\mathrm{CD} 8+\mathrm{T}$ cell activation is impaired in $\mathrm{CD}$ as well, and may help to explain the previous observations showing a defect in induction of CD8 $+\mathrm{T}$ cell proliferation by IEC derived from both CDI and CDU tissues (13). The reduced-to-absent upregulation of CD8-associated p56lck in this system correlates with the decrease in gp180 expression by these cells.

Mixed T cell-IEC coculture assays using 3G4 and 3G8 cells were also performed with IEC derived from a non-IBD inflammatory control specimen, DIV (Fig. 8). As in studies using
NL IEC, activation of p56lck was seen at $1 \mathrm{~min}$ in cocultures with $3 \mathrm{G} 8$ cells, but no phosphorylation was observed in the experiments with $3 \mathrm{G} 4$ cells. Thus, correlating with B9 staining, differences in p56lck phosphorylation patterns between NL and IBD IEC do not appear to be due to the state of inflammation per se.

\section{Discussion}

Previous studies from this laboratory have documented a defect in IEC stimulation of T cells in patients with IBD (10). In contrast to NL IEC, which stimulated CD8+ suppressor T cells in allogeneic mixed-cell cultures, IEC derived from both inflamed and uninflamed areas of IBD tissues activated potent CD4+ helper T cells. This difference in CD4+ versus CD8+ $\mathrm{T}$ cell activation did not appear to relate to enhanced class II antigen expression by these cells since other non-IBD inflammatory control IEC (ischemic colitis, gold-induced colitis, DIV) were perfectly capable of stimulating CD8+ T cells even in the face of comparable increases in MHC class II antigens. Thus, it appeared that there might be a fundamental defect in

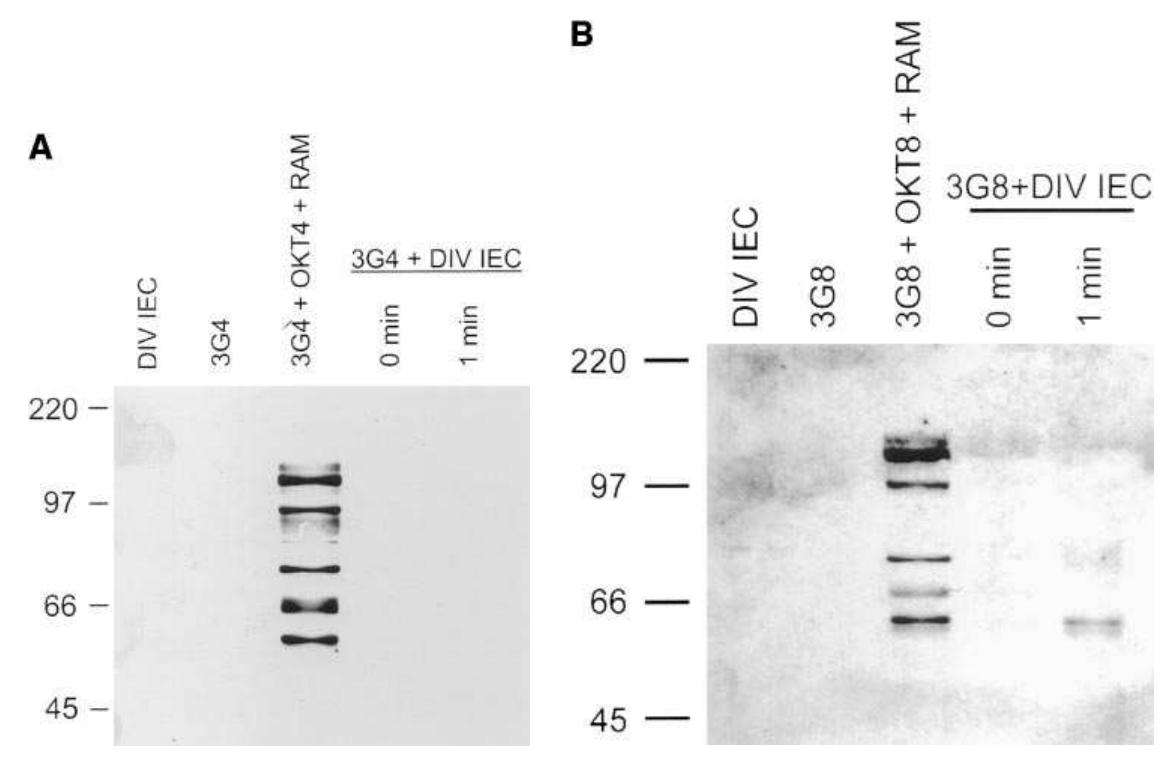

Figure 8. Antiphosphotyrosine Western blot analysis of cell lysates from mixed T cell-IEC cocultures. $(A) 3 \mathrm{G} 4$ or $(B) 3 \mathrm{G} 8$ cells were cocultured with IEC freshly isolated from DIV specimens for $0,1,2$, or $10 \mathrm{~min}$ (last two lanes not shown). Cell lysates were prepared, transferred to nitrocellulose membranes, and the membranes were treated as described in Fig. 6. Activation of p56lck was determined by enhanced chemiluminescence. Negative controls consisting of DIV IEC alone, 3G4 cells, and $3 \mathrm{G} 8$ cells are also shown. Positive controls showing p56lck activation when incubating $3 \mathrm{G} 4$ cells with anti-CD4 mAb (OKT4) and $3 \mathrm{G} 8$ cells with anti-CD8 $\mathrm{mAb}(O K T 8)$ are demonstrated as well. 
the ability of IBD IEC to activate suppressor T cells. We postulated that if IEC were functioning as nonprofessional antigen-presenting cells in the gut, antigens processed through IBD IEC would not only result in the failure to shut off mucosal immune responses but also participate actively in the immune activation reported in IBD by a number of groups.

We have identified recently two mAbs, B9 and L12, which inhibit selectively the activation of $\mathrm{CD} 8+\mathrm{T}$ cells by normal IEC (11). The mAbs B9 and L12 appear to recognize carbohydrate moieties on gp180, a molecule which has the ability to bind to CD8 and activate its associated kinase p56lck, an event essential for $\mathrm{T}$ cell activation in this system (12). If gp180 is responsible for $\mathrm{CD} 8+\mathrm{T}$ cell activation in the normal intestine, we postulated that the defect in $\mathrm{CD} 8+$ suppressor $\mathrm{T}$ cell activation by IBD IEC (14) might relate to differences in the expression of this molecule. Of note, we found that neither anticlass I nor anti-class II mAbs inhibit IEC-driven CD8+ T cell proliferation, suggesting that classical $\mathrm{MHC}$ antigens are not involved (13).

In this study, we demonstrate that mAbs B9 and L12 bind to apical and basolateral sites on surface and crypt IEC from NL and non-IBD inflammatory controls, but minimally stain CD IEC, and bind to UC IEC in a reduced and redistributed pattern. Thus, gp180 expression appears to be defective in IBD. Significantly, the abnormal staining patterns are observed in both inflamed and uninflamed areas of intestine, indicating that inflammation per se is not responsible for the differences seen. Indeed, inflammatory specimens from patients with DIV and ischemic colitis manifest a normal staining pattern. Furthermore, if anything, a reduction in inflammation correlates with a more striking decrease in gp180 expression in IBD tissues, as noted when comparing uninvolved areas with inflamed areas (Fig. 3). In addition, no correlation between medical regimen and gp180 expression was found. Hence, medication does not appear to play a role in the data described here. These findings, taken together with our previous observations, suggest that defects in gp180 expression may be intrinsic to IBD.

It is intriguing to note that the pattern of gp180 expression in the two diseases does not reflect the tissue distribution normally noted in these disorders (patchy expression in UC, where disease is more uniform, and diffusely decreased staining in $\mathrm{CD}$, where disease is more patchy). However, in both cases, the ability of gp180 to interact with T cells is reduced, since apically expressed gp180 is not likely to come in contact with $\mathrm{T}$ cells. Thus, both diseases may be functionally similar in terms of gp180-CD8 interactions. The differences in the pattern of disease expression are likely to be due to other factors, such as defects in the mucosal barrier (allowing for greater antigen access to IEC), luminal bacterial content, and underlying genetic predisposition. It has become increasingly apparent that both $\mathrm{CD}$ and UC are multigenic disorders. Some genes may be common to both diseases, while others provide the substrate for disease specificity. gp180 expression defects in IBD may have similar functional consequences with different genetic defects. The functional defect gives rise to the ability of IEC to activate CD4+ T cells while failing to simultaneously (or preferentially, as suggested in our DIV studies) activate CD8 $+\mathrm{T}$ cells.

In addition, we found that defects in gp180 expression by IBD IEC correlate with alterations in T cell activation by IEC. Our experiments with the transfectant cell lines 3G4 and 3G8 show that while normal IEC activate CD8- but not CD4-asso- ciated p56lck, IEC derived from both inflamed and uninflamed $\mathrm{UC}$ and CD preferentially activate CD4-associated p56lck. CD8 $\alpha$-associated $l c k$ and its substrates are also phosphorylated in T cell-IBD IEC cocultures, but to a lesser degree. These findings corroborate several pieces of evidence that strongly support a role for activated CD4+ $\mathrm{T}$ cells in the pathogenesis of IBD and the observation that, unlike normal IEC, IBD IEC activate CD4+ T cells but fail to stimulate CD8 + suppressor T cells $(9,10)$. Given that binding to gp180 appears to be essential to CD8+ suppressor T cell activation in $\mathrm{T}$ cell-IEC cocultures [in that antibodies to gp180 inhibit the activation of CD8-associated p56lck in T cell-IEC cocultures (13), and purified gp180 activates CD8+ T cells (12)], these data suggest that defects in gp180 may play a pathogenetic role in the IBD disease process by disrupting CD8 activation, leading to a failure of immune suppression. Activation of CD4+ T cells by IBD IEC can then occur unimpeded.

These findings provide a potential mechanism for the large number of studies documenting an increase in activated CD4+ $\mathrm{T}$ cells in IBD mucosa (21-25). These activated CD4+ T cells secrete cytokines that enhance the inflammatory state. Indeed, it has been suggested that the profile of cytokines produced by the CD4+ $\mathrm{T}$ cells may dictate the type of disease observed (type 1 for CD, and type 2 for UC) (3). Our data do not provide insights into selective $\mathrm{CD} 4+\mathrm{T}$ cell activation, although this, too, is likely to result from the genetic predisposition to mount type 1 versus type 2 responses to specific antigens. What our data do suggest is that luminal antigen (and it may be any luminal antigen, but more likely bacterial) sampled by IBD IEC will more likely present these antigens to CD4+ T cells. The nonselectivity of the inducing antigen may help to explain the inability to identify one specific bacterium or virus which is causative in these disorders.

Our work expands on the growing body of literature demonstrating that an interruption of normal $\mathrm{T}$ cell homeostasis is critical to the development of IBD. The development of colitis in IL-2, IL-10, and TGF- $\beta$ knockout mice highlights the importance of cytokine-mediated regulation of T lymphocytes (2628). Here, too, CD4+ T cells appear to play a significant role in the induction of colitis. Rudolphi and co-workers (29) have shown that intestinal CD4+ T cells from normal mice home preferentially to the intestinal mucosa and trigger disease upon transfer into severe combined immunodeficient (SCID) mice. Intriguingly, Powrie et al. (30-32) and Morrissey et al. (33) have defined specific subpopulations of normal CD4+ T cells, expressing high or low amounts of CD45RB, which have the capacity to induce or prevent colonic inflammatory disease, respectively, upon transfer into SCID mice. Moreover, investigators have discovered large numbers of CD4+ cells expressing low levels of CD45RB and L-selectin in IL-2 knockout mice (30-34). The development of colitis after bone marrow transplantation in $\operatorname{tg} \epsilon 26$ mice, where thymic development is distorted, is another example of dysregulated CD4+ T cell activity $(35,36)$. These studies, in addition to the CD45RB ${ }^{\text {hi }} / \mathrm{SCID}$ transfer model, underscore the critical nature of the absence or presence of regulatory $\mathrm{T}$ cells in the induction of disease. Our data support evidence demonstrating a strong role for CD4+ $\mathrm{T}$ cells and a disruption of regulatory $\mathrm{T}$ cell mechanisms in the pathogenesis of IBD.

The nature of the defect in gp180 expression in IBD IEC is not clear, but the pattern of immunohistochemical staining seen with mAbs B9 and L12 suggests that the defect is post- 
transcriptional. In UC IEC, there is a loss of basolateral staining, with preservation of gp180 expression at apical sites, intimating that the alteration is unlikely to be at the protein level, but occurs perhaps during posttranscriptional modification, causing a redistribution of the molecule. The differences in staining may also relate to altered glycosylation of gp180, since both $\mathrm{mAbs}$ B9 and L12 recognize N-linked carbohydrate epitopes (12). Further evidence supporting some posttranslational defect in both UC and CD IEC is that gp180 can be upregulated, as shown by our studies demonstrating upregulation in inflamed versus uninflamed IBD IEC and upregulation of gp180 expression by INF- $\gamma$ and GM-CSF in epithelial cell lines (20). However, the decrease in staining with mAb B9 or L12 on CD IEC at both apical and basolateral sites suggests that the defect in CD is distinct from that found in UC.

In summary, a more global defect in gp180 expression in CD IEC would explain the failure of these cells to activate CD8+ suppressor T cells. For UC IEC, where gp180 expression remains partially intact, the explanation is less clear. However, the absence of normal gp180 expression on UC IEC at basolateral sites, where interactions with lamina propria lymphocytes and intraepithelial lymphocytes are most likely to occur in vivo, may be one mechanism by which UC IEC fail to activate suppressor cells in the host. Regardless, these findings may help to explain the uncontrolled inflammation seen in IBD. In this model, presumably any luminal antigen might trigger the initial immune response through the activation of CD4+ T cells, and the failure of gp180 to activate CD8+ T cells explains its perpetuation.

\section{Acknowledgments}

We would like to thank Drs. Adrian Greenstein, Joel Bauer, Thomas Heimann, Isadore Kreel, Noam Harpaz, and Randall Steinhagen, as well as the late Dr. Irwin Gelernt, who graciously provided many of the specimens utilized in these studies.

This work was supported by United States Public Health Service grants AI23504, AI24671, and DK44156 (L. Mayer), a Crohn's and Colitis Foundation of America Research Fellowship Award, a grant from the Burrill B. Crohn Research Foundation, and the KennedyLeigh Charitable Trust.

\section{References}

1. Zeitz, M., R. Ullrich, H.L. Schieferdecker, A.N. Weiss-Breckwoldt, S.P. James, and E.O. Riecken. 1991. Characterization of T cell subpopulations in the intestinal lamina propria in inflammatory bowel disease. In Inflammatory Bowel Diseases: Progress in Basic Research and Clinical Implications. H. Goebell, K. Ewe, H. Malchow, and C. Koelbel, editors. Kluwer Academic Publishers, Hingham, MA. 63-70.

2. Mueller, C., P. Knoflach, and C.C. Zielinski. 1990. T cell activation in Crohn's disease. Increased levels of soluble interleukin-2 receptor in serum and in supernatants of stimulated peripheral blood mononuclear cells. Gastroenterology. 98:639-646.

3. Mullin, G.E., A.J. Lazenby, M.L. Harris, T.M. Bayless, and S.P. James. 1992. Increased interleukin-2 messenger RNA in the intestinal lesions of Crohn's disease but not ulcerative colitis. Gastroenterology. 102:1620-1627.

4. Breese, E., C.P. Braegger, C.J. Corrigan, J.A. Walker-Smith, and T.T. MacDonald. 1993. Interleukin-2- and interferon-gamma-secreting $\mathrm{T}$ cells in normal and diseased human intestinal mucosa. Immunology. 78:127-131.

5. Breese, E.J., C.A. Michie, S.W. Nicholls, S.H. Murch, C.B. Williams, P. Domizio, J.A. Walker-Smith, and T.T. MacDonald. 1994. Tumor necrosis factor alpha-producing cells in the intestinal mucosa of children with inflammatory bowel disease. Gastroenterology. 106:1455-1466.

6. Sartor, R.B. 1994. Cytokines in intestinal inflammation: pathophysiological and clinical considerations. Gastroenterology. 106:533-539.

7. Halstensen, T.S., T.E. Mollnes, and P. Brandtzaeg. 1989. Persistent complement activation in submucosal blood vessels of active inflammatory bowel disease: immunohistochemical evidence. Gastroenterology. 97:10-19.

8. Halstensen, T.S., T.E. Mollnes, O. Fausa, and P. Brandtzaeg. 1989. Deposits of terminal complement complex (TCC) in muscular mucosa and submucosal vessels in ulcerative colitis and Crohn's disease of the colon. Gut. 30:361-366.

9. Bland, P.W., and L.G. Warren. 1986. Antigen presentation by epithelial cells of the rat small intestine. I. Selective induction of suppressor T cells. Immunology. 58:9-14.

10. Mayer, L., and R. Shlien. 1987. Evidence for function of Ia molecules on gut epithelial cells in man. J. Exp. Med. 166:1471-1483.

11. Mayer, L., E. Siden, S. Becker, and D. Eisenhardt. 1990. Antigen handling in the intestine mediated by normal enterocytes. In Advances in Mucosal Immunology. T.T. MacDonald, S.J. Challacombe, P.W. Bland, C.R. Stokes, R.V. Heatley, and A.M. Mowat, editors. Kluwer Academic Publishers, Norwell, MA. 23-28.

12. Yio, X.Y., and L. Mayer. 1997. Characterization of a 180-kDa intestinal epithelial cell membrane glycoprotein, gp180. A candidate molecule mediating T cell-epithelial cell interactions. J. Biol. Chem. 272:12786-12792.

13. Li, Y., X.Y. Yio, and L. Mayer. 1995. Human intestinal epithelial cellinduced CD8 $+\mathrm{T}$ cell activation is mediated through CD8 and the activation of CD8-associated p56lck. J. Exp. Med. 182:1079-1088.

14. Mayer, L., and D. Eisenhardt. 1990. Lack of induction of suppressor T cells by intestinal epithelial cells from patients with inflammatory bowel disease. J. Clin. Invest. 86:1255-1260.

15. Mayer, L., D. Eisenhardt, P. Salomon, W. Bauer, R. Plous, and L. Piccinini. 1991. Expression of class II molecules on intestinal epithelial cells in humans: differences between normal and inflammatory bowel disease. Gastroenterology. 100:3-12.

16. Panja, A., A. Barone, and L. Mayer. 1994. Stimulation of lamina propria lymphocytes by intestinal epithelial cells: evidence for recognition of non-classical restriction elements. J. Exp. Med. 179:943-950.

17. Holmes, K.H., and B.J. Fowlkes. 1991. Labeling antibody with fluorescein isothiocyanate (FITC). In Current Protocols in Immunology. J.E. Coligan, A.M. Kruisbeek, D.H. Margulies, E.M. Shevach, and W. Strober, editors Greene Publishing Associates and Wiley-Interscience, New York. 5.3.2-5.3.3.

18. Sleckman, B.P., A. Peterson, W.K. Jones, J.A. Foran, J.L. Greenstein, B. Seed, and S.J. Burakoff. 1987. Expression and function of CD4 in a murine T-cell hybridoma. Nature (Lond.). 328:351-353.

19. Ratnofsky, S.E., A. Peterson, J.L. Greenstein, and S.J. Burakoff. 1987. Expression and function of CD8 in a murine T cell hybridoma. J. Exp. Med. 166:1747-1757.

20. Campbell, N.A., X. Yio, L. Toy, and L. Mayer. 1996. Cytokine regulation of gp180, a CD8 ligand expressed by intestinal epithelial cells. Gastroenterology. 110:A876. (Abstr.)

21. Fais, S., F. Pallone, O. Squarcia, M. Boirivant, and P. Pozzilli. 1985. T cell early activation antigens expressed by peripheral lymphocytes in Crohn's disease. J. Clin. Lab. Immunol. 16:75-76.

22. Strickland, R.G., S. Korsmeyer, R.D. Soltis, I.D. Wilson, and R.C. Williams. 1974. Peripheral blood T and B cells in chronic inflammatory bowel disease. Gastroenterology. 67:569-577.

23. Mueller, C., P. Knoflach, and C.C. Zielinski. 1990. T cell activation in Crohn's disease: increased levels of soluble interleukin-2 receptor in serum and in serum supernatants of stimulated peripheral blood mononuclear cells. Gastroenterology. 98:639-646.

24. Pallone, F., S. Fais, O. Squarcia, L. Biancone, P. Pozzilli, and M. Boirivant. 1987. Activation of peripheral blood and intestinal lamina propria lymphocytes in Crohn's disease. In vivo state of activation and in vitro response to stimulation as defined by the expression of early activation antigens. Gut. 28: $745-753$.

25. Raedler, A., S. Fraenkel, G. Klose, and H.G. Thiele. 1985. Elevated numbers of peripheral $\mathrm{T}$ cells in inflammatory bowel diseases displaying $\mathrm{T} 9$ antigen and Fc alpha receptors. Clin. Exp. Immunol. 60:518-524.

26. Sadlack, B., H. Merz, H. Schorle, A. Schimpl, A.C. Feller, and I. Horak. 1993. Ulcerative colitis-like disease in mice with a disrupted interleukin-2 gene. Cell. 75:253-261.

27. Kuhn, R., J. Lohler, D. Rennick, K. Rajewski, and W. Muller. 1993. Interleukin-10-deficient mice develop chronic enterocolitis. Cell. 75:263-274.

28. Shull, M.M., I. Ormsby, A.B. Kier, S. Pawlowski, R.J. Diebold, M. Yin, R. Allen, C. Sidman, G. Proetzel, D. Calvin, et al. 1992. Targeted disruption of the mouse growth factor b1 gene results in multi-focal inflammatory disease. Nature (Lond.). 359:693-699.

29. Rudolphi, A., G. Boll, S.S. Poulsen, M.H. Claesson, and J. Reimann. 1994. Gut homing CD4+ T cell receptor ab $+\mathrm{T}$ cells in the pathogenesis of murine inflammatory bowel disease. Eur. J. Immunol. 24:2803-2812.

30. Powrie, F., M.W. Leach, S. Mauze, L.B. Caddle, and R.L. Coffman. 1993. Phenotypically distinct subsets of CD4+ T cells induce or protect from chronic intestinal inflammation in C.B-17 SCID mice. Int. Immunol. 5:14611471.

31. Powrie, F., R. Correa-Oliveira, S. Mauze, and R.L. Coffman. 1994. Regulatory interactions between $C D 45 \mathrm{RB}^{\text {high }}$ and $\mathrm{CD} 45 \mathrm{RB}^{\text {low }} \mathrm{CD} 4+\mathrm{T}$ cells are important for the balance between protective and pathogenic cell-mediated immunity. J. Exp. Med. 179:589-600.

32. Powrie, F., M.W. Leach, S. Mauze, S. Menon, L.B. Caddle, and R.L. 
Coffman. 1994. Inhibition of TH1 responses prevents inflammatory bowel disease in SCID mice reconstituted with CD45RB hi $C D 4+T$ cells. Immunity. $1: 553-562$.

33. Morrissey, P.J., K. Charrier, S. Braddy, D. Liggitt, and J.D. Watson. 1993. CD4+ T cells that express high levels of CD45RB induce wasting disease when transferred into congenic severe combined immunodeficient mice. Disease development is prevented by co-transfer of purified CD4+ T cells. J. Exp. Med. 178:237-244.

34. Simpson, S.J., E. Mizoguchi, D. Allen, A.K. Bhan, and C. Terhorst.
1995. Evidence that CD4+ but not CD8 + T cells are responsible for murine interleukin-2-deficient colitis. Eur. J. Immunol. 25:2618-2625.

35. Hollander, G.A., B. Wang, A. Nichogiannopoulou, P.-P. Platenberg, W. van Ewijk, S.J. Burakoff, J.-C. Gutierrez-Ramos, and C. Terhorst. 1995. Developmental control point in induction of thymic cortex regulated by a subpopulation of prothymocytes. Nature (Lond.). 373:350-353.

36. Hollander, G.A., S.J. Simpson, E. Mizoguchi, A. Nichogiannopoulou, J. She, J.-C. Gutierrez-Ramos, A. Bhan, S.J. Burakoff, B. Wang, and C. Terhorst. 1995. Severe colitis in mice with aberrant thymic selection. Immunity. 3:27-38. 